Physics Vol. 3, No. 5, pp. 255-283, 1967. Physics Publishing Co. Printed in Great Britain.

\title{
ThE THEORY OF CONDENSATION AND THE CRITICAL POINT
}

\author{
MICHAEL E. FISHER \\ Baker Laboratory, Cornell University, Ithaca \\ New York 14850 \\ (Received 27 March 1967)
}

\begin{abstract}
The droplet or cluster theory of condensation is reviewed critically and extended. It is shown to imply that the condensation point is marked by a singularity of the thermodynamic potential as conjectured by Mayer. The singularity turns out to be an essential singularity at which all derivatives of the thermodynamic variables remain finite. The theory also yields an understanding of the uniqueness of the critical point (in contrast to an extended critical region or Derby-hat type of behaviour) and leads to relations between the various critical point singularities.

A one-dimensional model is described with a Hamiltonian containing short-range many-body potentials. The exact solution of the model is sketched and shown to exhibit condensation and critical phenomena for suitable (fixed) potentials. The analysis confirms the conclusions of the cluster theory and thereby lends support to the validity of its underlying assumptions.
\end{abstract}

\section{Introduction}

IN THIS lecture* I will review some old ideas concerning the theory of condensation and will describe some new work and new conclusions that may also throw light on the nature of the critical region. To introduce our topic let us pick out some of the highlights in the historical development.

- This article is the text of a lecture given at the Centennial Conference on Phase Transformation held at the University of Kentucky, Lexington, Kentucky, 18th-20th March 1965 . It is reproduced here essentially in the same form as presented and later circulated privately except for the addition of Appendix B. Attention should be drawn to the following more recent articles: (a) as regards the condensation point; J.S. LANGER, Ann. Phys. (N.Y.), 41, 108 (1967); (b) concerning critical point singularities, B. WIDOM, J. Chem. Phys. 43, 3892,3898 (1965); C. DOMB and D.L. HUNTER, Proc. phys. Soc. 86, 1147 (1965); L.P. KADANOFF, Physics 2, 263 (1966); A.Z. PATASHINSKII and V.L. POKROVSKII, Soviet Phys. JETP 23, 292 (1966); (c) in regard to related types of one-dimensional model: L.K. RUNNELS, J. Chem. Phys. 42,212 (1965); D. POLAND and H. SCherAgA, J. Chem. Phys. 45, 1464 (1966); M.E. FISHER, J. Chem. Phys. 45, 1469 (1966). 
Onsager by his exact solution of the two-dimensional Ising model over twenty years ago [1] 'demonstrated clearly how a phase transition would come out of statistical mechanics alone if only one were clever enough to compute the partition function precisely: there is no need of any additional assumptions, special procedures or the like. This is the philosophy to which I will adhere throughout, namely, to describe the equilibrium properties of a physical system we need only calculate the partition function and use the formalism of statistical mehcanics.

In 1952 Yang and Lee [2], showed how the discontinuities and related mathematical singularities which characterize the thermodynamic potentials of systems exhibiting phase transitions could "grow" from the completely smooth and analytic partition functions of finite systems. This occurs, of course, only when one proceeds to the "thermodynamic limit" in which the volume of the system becomes infinite while the intensive variables remain finite. The analysis of Lee and Yang is very general and does not therefore reveal the detailed nature of the singularities to be expected in any particular type of phase transition.

More recently Kac, Uhlenbeck and Hemmer [3], have shown how the old ideas of van der Waals give some answers to the question of the nature of the condensation singularities, at least in the case of a system of particles interacting with very weak, long range attractive forces (in addition to the ever present strong short range repulsive forces). More precisely the van der Waals description of the condensation of a fluid becomes rigorously correct if the limit of infinitely long-range and infinitely weak attractive pair interaction forces is taken after the thermodynamic limit $[3,4]$. While it adds appreciably to our understanding of phase transitions, this conclusion is not entirely satisfying since the systems in which one sees condensation phenomena experimentally are characterized, in the main, by attractive forces of quite short range. One suspects (and indeed the soluble one-dimensional examples tell us) that the behaviour of such systems may be appreciably different.

This observation forms the point of departure of the present discussion. We will endeavour to discover the type of analytic behaviour which occurs in the neighbourhood of a condensation point and will conclude that is probably quite different, and much more subtle, than the classical van der Waals description would suggest. I will also have something to say about the nature and uniqueness of the critical point which marks the limit of the condensation points. Our theme will thus be one of "hunt the singularity"!

\section{Mayer's Conjecture}

Experimentally one observes that a system of molecules interacting through short range repulsive forces (giving the molecules an essentially incompressible "core") and short range attractive forces, will undergo an abrupt transformation from a gaseous to a liquid state (or to a solid state at low enough temperatures) even at very low (gaseous) densities. It is this low density condensation phenomena occurring well below the critical temperature, which we shall consider. It is significant, furthermore, that the isothermal compressibility of the gas

$$
K_{T}=\rho^{-1}(\partial \rho / \partial p) T
$$

is observed to remain finite as the condensation point $\rho=\rho_{\sigma}$ is approached (see Fig. 1). The symbols $p, p$ and $T$ denote, as usual, the pressure, density and temperature.

If the system is allowed to go into a non-equilibrium state one can also find experimentally a metastable "continuation" of the gaseous isotherm into a region describing a supercooled 
vapour (see Fig. 1). Although, when suitable experimental precautions are taken, this isotherm

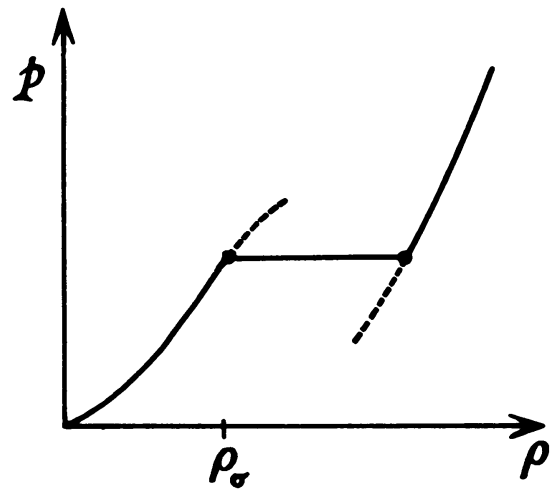

FIGURE 1

An isotherm illustrating condensation in a real system.

can be quite prolonged and reproducible it must be stressed that the system is no longer in a state of complete thermodynamic equilibrium. Furthermore it may be shown rigorously that such nonequilibrium metastable states cannot be found in a correct statistical mechanical calculation based on the total partition function for the system [5]. (If they are found the calculation has been incorrect!)

As is well known the van der Waals, and equivalent theories of condensation yield an isotherm with a "loop" (as shown in Fig. 2) that continues analytically through the condensation region

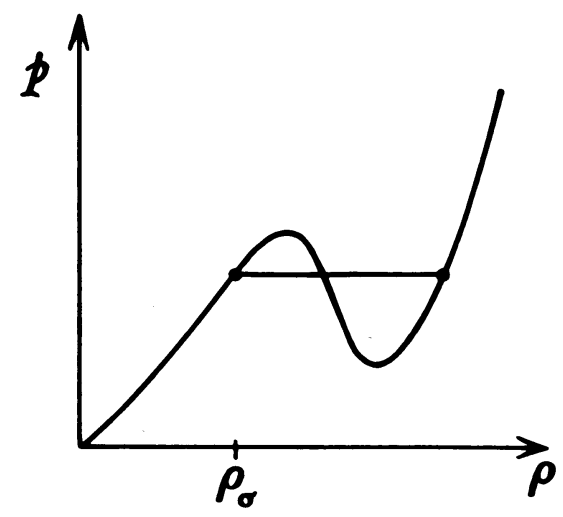

FIGURE 2

Condensation in a van der Waals system.

and even includes an "unstable" portion with negative compressibility. The correct equilibrium isotherm must be found by a Maxwell, or better, Gibbs construction which yields the horizontal 
"two-phase" portion of the isotherm so "cutting off" the "metastable" and "unstable" portions of the loop. (This construction comes automatically out of the rigorous Kac, Uhlenbeck and Hemmer theory $[3,4]$.

In consequence the gaseous isotherm remains perfectly smooth and analytic as the condensation point is approached: there is no singularity (in the mathematical sense of a nonanalytic point) on the isotherm to "warn" of the onset of condensation. On the contrary the isotherm may be analytically continued to larger densities to yield the original looped isotherm. (The fact that the van der Waals type of theory describes a "metastable" continuation of the isotherm in this way is sometimes considered to be an advantage of the theory; in my opinion, however, the comments made above indicate that this is unjustified.)

The converse suggestion, namely, that the gaseous isotherm should exhibit some sort of mathematical singularity at the condensation point, which could thus be located even if the two-phase and liquid parts of the isotherm were unknown, was made by Mayer [6]. He based his arguments on the fundamental expansions

$$
\begin{aligned}
& p / k T=\pi(z)=\sum_{l=1}^{\infty} b_{l} z^{l} . \\
& \rho=z \frac{\partial \pi}{\partial z}=\sum_{l=1}^{\infty} l b_{l} z^{l} .
\end{aligned}
$$

and on the related virial expansion

$$
\text { - } p / k T=\sum_{i=1}^{\infty} \beta_{l p^{l}} .
$$

obtained by eliminating the activity $z$. Mayer tried to analyse the form of the cluster coefficients $b_{l}$ for large $l$ since one knows from the theory of analytic functions that this determines the nature of the singularities of the function. The radius of convergence of a power series such as (2) is determined by the behaviour of the coefficients for large $l$ through

$$
r_{0}=\left.\lim _{l \rightarrow \infty} \inf _{l \rightarrow}\right|^{-1 / l}
$$

On the circle of convergence $|z|=r_{0}$, there must be at least one singularity $z_{0}$ of the function $\pi(z)$ and no other singularities in the complex plane can lie closer to the origin. Furthermore if the coefficients are of uniform sign for large $l$ the dominant singularity $z_{0}$ lies on the positive real axis.

In its strong form we may express Mayer's conjecture by the assertion that a closest singularity $z=z_{0}$ of $\pi(z)$ will lie on the positive real axis and will occur at the condensation point $z=z_{\sigma}$. In this form the conjecture is quite possibly wrong in most realistic cases, since accumulating evidence suggests that the $b_{l}$ will oscillate in sign for large $l$ (essentially owing to the repulsive cores of the molecular interactions) so that the singularity $z_{0}$ determining the circle of convergence will lie on the negative real axis (i.e. in an unphysical region). 
I want to consider, however, the more general form of the conjecture which asserts merely that the function $\pi(z)$, defined by the power series (2) and its analytic continuation, has, on the real axis, a nearest singularity $z=z_{1}$ which occurs at the condensation point $z=z_{\sigma}$. We might then have $z_{1}>\left|z_{0}\right|$. so that $z_{1}$ lies outside the circle of convergence of (2), but since the series may be analytically continued along the real axis up to $z_{1}$ this does not matter.

Of course, the van der Waals theory constitutes a counterexample to this conjecture since the nearest singularity on the real $z$ axis occurs beyond the condensation point i.e. $z_{1}>z_{\sigma}$. In fact the value of $z_{1}$ corresponds to the first point of infinite compressibility on the analytically continued isotherm (which is sometimes termed the "limit of metastability of the gaseous phase"). In a review of this question Katsura [7] has argued that this should be the general case. As we have pointed out, however, the van der Waals and similar theories cannot be taken as guides on this point owing to the unrealistic nature of the interactions they imply.

Another argument sometimes used suggests that the question of a singularity at $z=z_{\sigma}$ in the activity series is an artificial one since, it is asserted, the virial series might have no singularity at the corresponding condensation point $\rho=\rho_{\sigma}$ so that the singularity at $z_{\sigma}$ could be of no physical significance. As an example of this the ideal Bose-Einstein gas is cited. In that case the activity series has a singularity at the Bose-Einstein condensation point (i.e. $z_{1}=z_{\sigma}$ ) but the virial series is quite analytic at the corresponding density (and the isotherm can be continued on to higher densities). When one looks more closely, however, one finds that this example is also artificial because of the special shape of the isotherm near condensation. At fixed $T$ this is given by

$$
p=p_{\sigma}-A\left(p_{\sigma}-\rho\right)^{2}+\ldots
$$

which means that the isothermal compressibility becomes infinite as the condensation point is approached (see Fig. 3).

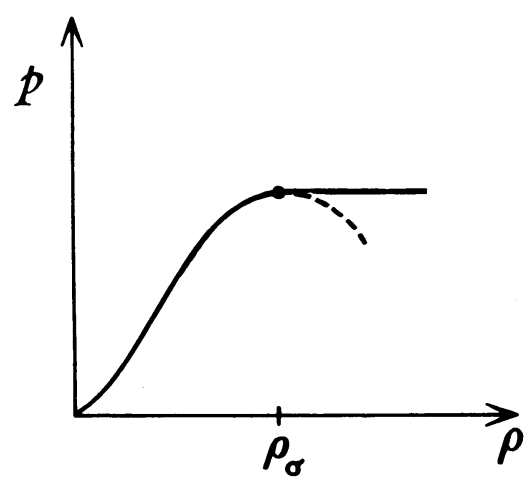

FIGURE 3

\section{Condensation in an ideal Bose-Einstein fluid.}

This feature is, of course, not found in real condensation processes. Conversely one may prove quite easily (see Appendix A) that if at fixed temperature the pressure $p(p)$ is analytic in the density at $p=p_{\sigma}$ and if 


$$
K_{T}\left(\rho_{\sigma-}\right)=\lim _{\rho \rightarrow \rho_{\sigma^{-}}} K_{T}(\rho)<\infty
$$

then the pressure $\bar{p}(z)$ is also analytic in the activity at $z=z_{\sigma}$. This shows that if the compressibility remains finite as a condensation point is approached, then a singularity in the activity series necessarily implies one in the virial series. Thus one cannot "escape" a singularity and hence it presumably has some physical significance.

\section{Surface Tension and the Droplet Model}

When we ask for the nature of the condensation singularities in a system with predominantly short range forces we are hampered because, apart from some examples I will describe later, there are no known model systems which are exactly soluble and exhibit condensation. It is true that we know rigorously that a two-dimensional Ising system with nearest neighbour forces undergoes condensation but since Onsager's solution is restricted, in "magnetic language", to zero field, that is in "fluid language" to $z=z_{\sigma}(T)$, one does not know what happens as the condensation point is approached. One may get a physical idea as to the "cause" of condensation, however, by considering a real gas or, for that matter, a lattice gas or Ising model, at low densities and temperatures. Evidently most configurations of the system will consist of distributions of isolated molecules well separated from one another. There will also be present, however, clusters of two, three or more molecules bound together more-or-less tightly by the attractive forces but isolated, for the most part, from other clusters. Clusters of different sizes will be in mutual statistical equilibrium, associating and disassociating, but even fairly large clusters resembling "droplets" of the liquid phase will have some, generally rather small, chance of occurring.

Consider the potential energy $E_{l}$ of such a not too small cluster of $l$ molecules. This may be decomposed into a bulk term, determined by the binding energy per molecule in the liquid or condensed phase, say $E_{0}$, and a remainder $W$ which is, evidently, associated with the loss of binding energy at the "surface" of the cluster. Thus

$$
E_{l}=l E_{0}+W \text {. }
$$

where $W$ is positive and may be taken as proportional to a "suitably defined" surface area $s$ of the cluster, that is

$$
W \simeq w s .
$$

For a lattice gas with nearest neighbour attractive forces the surface $s$ may be defined unambiguously in terms of the numbers of "unsaturated bonds" surrounding the cluster (that is bonds between occupied and unoccupied sites) and the surface energy density $w$ is similarly well defined. More generally, however, it is difficult to define $s$ precisely in a way that is not somewhat arbitrary. Nevertheless for a large enough cluster (which is not too "drawn out" like a piece of seaweed for example!) the surface area is faily well defined.

Now it is clear that the surface of a cluster gives it stability; because the surface energy is positive the cluster will, at low temperatures, tend to stay in compact configurations with relatively small surfaces. This teniency to shrink will, speaking loosely, be opposed by the entropy of the cluster which might similarly be expected to be of the form

$$
S_{l}=l S_{0}+\omega s
$$


where $S_{0}$ is the entropy per molecule in the bulk fluid. The surface entropy density $\omega$ is a measure of the number of different configurations of the cluster which have the same surface area. If now the temperature is lowered (or, what is essentially equivalent, if the activity is raised) the entropy will be less important and it becomes advantageous for clusters to combine to form droplets and for droplets to grow further by amalgamation thereby reducing the total surface area and hence lowering the total energy. Indeed if conditions are sufficiently favourable the droplets should continue to grow rapidly to macroscopic size. A macroscopic droplet represents, of course, the liquid phase and so its presence indicates that condensation has taken place!

This picture of the condensation of a gas was put forward apparentlv quite independently by Frenkel, Band and Bijl some twenty-seven years ago [8-10j. Bijl's work was the earliest, being contained in his thesis presented in April 1938, but it was not otherwise published. The papers of Frenkel and Band both appeared early in 1939 in The Journal of Chemical Physics. Although the ideas are quite straightforward and would have been understood many years previously, the stimulus in all three cases seems to have come from Mayer's work on the activity and virial expansions where the coefficients are determined by the famous cluster integrals. (In Mayer's theory, however, the "clusters" cannot be identified directly with real physical clusters as in the droplet picture. This seems to be the cost of making a fully rigorous and complete expansion.)

The essential correctness of the droplet explanation of the origin of condensation in a system with short range forces may be seen from the discussion of the existence of spontaneous magnetization in the plane Ising model ferromagnet given already in 1936 by Peierls [11]. The ideas of the surface tension and the entropy of clusters (in this case of 'overturned spins') play a crucial part in his argument although they are not expressed explicitly in this language. More recently R.B. Griffiths has adapted Peierls analysis to give a fully rigorous proof that condensation takes place in the Ising model [12]. The nature of the singularity, however, is still unrevealed. Furthermore the proof uses in an apparently essential way the symmetry of the Ising model under a change of sign of the field. Unfortunately more general models do not have such an exact symmetry although experimentally the shape of coexistence curves indicates a fairly precise symmetry between simple liquids and their vapours.

To clothe the droplet or cluster theory in mathematical form and to explore its consequences - and its difficulties - let us construct the classical configurational partition function for a cluster of $l$ molecules in a domain of volume $V$. We have

$$
q_{l}(\beta, V)=\frac{1}{l !} \int d \mathbf{r}_{1} \ldots \int d \mathbf{r}_{l} \exp \left(-\beta U_{l}\right)
$$

where $U_{l}=E_{l}$ is the potential energy of interaction and where the integrations are restricted to configurations in which the $l$ molecules form a cluster. For definiteness we may suppose the pair interaction potential $\phi(r)$ has an attractive tail of range $b$ so that $\phi(r) \equiv 0$ for $r \geqslant b$ and then define a "clustering distance" $c$ so that if $\left|\mathbf{r}_{i}-\mathbf{r}_{j}\right|<c$ the $i$-th and $j-$ th molecules belong to the same cluster. It is, of course, natural to take $c=b$. We will simplify further by assuming the potential has an infinite hard core and an attractive square well, that is

$$
\begin{array}{rlrl}
\phi(r) & =\infty \quad \text { for } r<a, \\
& =-\varphi_{0}, & & a \leqslant r<b \\
& =0, & & r \geqslant b .
\end{array}
$$


In this case the energy $E_{l}$ of any configuration is an integral multiple of - $\Phi_{0}$. Furthermore if $b / a$ is not too large the binding energy in the dense phase in three dimensions will be $F_{0}=$ $1 / 2\left(12 \varphi_{0}\right)=6 \Phi_{0}$ since hard spheres will pack with twelve nearest neighbours. The surface energy $W$ may then be defined precisely through (8). Taking $w=\phi_{0}$ so that $W=\phi_{0} s$ then defines the surface "area" $s$ and shows it is an integer as in the lattice case. Inserting these relations in (11) we may write

$$
q_{l(\beta, V)}=V \sum_{s} g(l, s) e^{l \beta E_{0}-\beta_{w s}}
$$

where the combinatorial factor $g(l, s)$ is the number (or more correctly the volume in $d(l-1)$ dimensional configuration space) of configurations of $l$ indistinguishable molecules with a fixed centre of mass which form a cluster of surface area $s$. (For a lattice gas, of course, the configurations are discrete; conversely in a more general model one would have an integration over a continuous range of $s$.) The factor $V$ comes from the integration of one coordinate over the volume with the neglect of boundary effects.

Following De Boer's treatment [13] consider now the coefficient of $z^{N}$ in

$$
\begin{aligned}
\mathscr{S} & =\exp \left[\sum_{l=1}^{\infty} q_{l} z^{l}\right] \\
& =1+\frac{1}{1 !} \sum_{l=1}^{\infty} q_{l} z^{l}+\frac{1}{2 !}\left[\sum_{l=1}^{\infty} q_{l} z^{l}\right]^{2}+\ldots
\end{aligned}
$$

Evidently this coefficient consists of the sums of products of cluster partition functions formed by decomposing $N$ identical molecules into clusters in all possible ways. If we now assume that:

(A) the effects of excluded volume between clusters may be neglected, then this coefficient is simply the total configurational partition function $Q_{N}(\beta, V$. Thus

$$
\mathscr{S}=\sum_{N=0}^{\infty} z^{N} Q_{N}(\beta, V)
$$

which is recognised as the grand partition function $\equiv(z, \beta ; \eta$ for the system. For a large system the pressure is given in the standard way, by

$$
\begin{aligned}
p / k T & =(1 / V) \text { ln } \equiv(z, \beta ; V) \\
& =\sum_{l=1}\left(q_{l} / V\right) z^{l}
\end{aligned}
$$

provided the series converges (see below), and hence the density is 


$$
\rho=\langle N\rangle / V=\sum_{l=1}^{\infty} l\left(q_{l} / V\right) z^{l} .
$$

One may readily check (for example by introducing separate activity coefficients $z_{l}$ for clusters of size $l$ ) that the partial number densities of $l$-clusters, which are proportional to the probability of finding an $l$-cluster, are just

$$
p_{l}=\left(q_{l} / V\right) z^{l} .
$$

By neglecting the interaction, that is essentially the excluded volume, between clusters the calculation of the equation of state has thus been reduced to the calculation of the singlecluster 'internal partition function', $q_{l} / V$. One feels that at low densities the excluded volume effects should not be important (although nearer the critical point they might be). Nevertheless the assumption (A) is obviously an important and potentially far reaching one and we will return to it later. To proceed further, however, the cluster partition function must be analysed in greater detail.

\section{Cluster Partition Function and Condensation}

Even for a lattice gas a full analysis of the combinatorial factor $g(l, s)$ entering in (13) seems very difficult. This is an important theoretical task central, as we will see, to the study of condensation. To see where the essential difficulties lie let us put

$$
\begin{aligned}
G_{l}(\beta) & =\sum_{s} g(l, s) e^{-\beta w s} \\
& =e^{-l \beta E_{0}} q_{l}(\beta, V) / V
\end{aligned}
$$

and observe firstly that the bulk entropy per particle $S_{0}$ in a large cluster may be defined by

$$
S_{0}(\beta)=k \lim _{l \rightarrow \infty}(1 / l) \ln G_{l}(\beta) \text {. }
$$

The existence of the limit in (21) can be proved rigorously by a generalised subadditive argument based on decomposing a cluster of $l$ particles into two clusters of $l^{\prime}$ and $l-l^{\prime}+1$ particles with one particle in common. This justifies the assumption of a bulk contribution to the entropy.

Secondly notice that the surface of a cluster cannot exceed some constant multiple of the number of particles (as is achieved by a cluster in the form of a string of beads, for example) nor can it be less than some minimum surface attained by some approximately spherical cluster. In $d$ dimensions we thus have

$$
a_{1} l^{1-(1 / d)} \leqslant s \leqslant a_{2} l .
$$

where $a_{1}$ and $a_{2}$ are appropriate constants. Since the terms in (19) are all positive the standdard maximum term argument shows that 


$$
\max _{s}\left\{g(l, s) e^{-\beta w s}\right\} \leqslant\left(G_{l} \beta\right) \leqslant a_{2} l \max _{s}\left\{g(l, s) e^{-\beta w s}\right\}
$$

Suppose that the maximum is attained for $s=\bar{s}$ so that $\bar{s}=\bar{s}(l ; \beta)$ is the most probable or, loosely, mean surface area. Then as $l$ becomes large

$$
\ln G_{l}(\beta)=\ln g[l, \bar{s}(l ; \beta)]-\beta w \bar{s}(l ; \beta)+O(\ln l) .
$$

For the present we now avoid the full weight of the combinatorial problem by arguing that at low temperatures the most important configurations will be those relatively compact, roughly globular arrangements which have surface areas not vastly greater than the minimum possible and hence, for large $l$, increasing more slowly than $l$. We thus assume

(B i) the mean surface area $\bar{s}(l, \beta)$ satisfies

$$
\bar{s}(l ; \beta) / l \rightarrow 0 \quad \text { as } l \rightarrow \infty \text {. }
$$

which is almost tantemount to the meaning of any well defined "surface". By virtue of the lower limit in (22) we may also assume that

$$
\bar{s}(l ; \beta) / \ln l \rightarrow \infty \text { as } l \rightarrow \infty \text {. }
$$

These two assumptions are all that is essential for the main conclusions of the theory but in view of the bounds (22) and what has been said it is natural to expect more specifically that

$$
\bar{s}(l, \beta) \approx a_{0} l \sigma \quad(l \rightarrow \infty)
$$

with $a_{0}=a_{0}(\beta)$ and $\sigma=\sigma(\beta)$ satisfying

$$
0<\sigma<1 \text {. }
$$

In particular at low temperatures one might expect the exponent $\sigma$ to be equal to (or close to) the value $2 / 3$ for $d=3$ while for $d=2$ it should be $1 / 2$. These specific assumptions were in fact made by the early workers. It seems quite possible however, that an "effective mean surface area " which took account of the interference between clusters at finite densities due to the excluded volume might lead to a smaller exponent. Conversely the vastly larger number of configurations which can occur with a larger area might tend to increase the value of $\sigma$ [14]. Consequently even if we accept (27) and (28) as a convenient and concrete expression of the basic assumptions (25) and (26), it seems better to leave the value of the exponent $\sigma$ as an open question (but see further below).

From (21), (24) and (25) we see that $k \ln g[l, \bar{s}(l)]$ varies as $l S_{0}$ for large $l$ so that the difference defines a residual or "surface entropy". In fact this entropy will be associated directly with the many possible configurations of a section of the surface of a cluster (large in itself but small compared with the total surface). It is thus natural to assume finally that

(B iii) the residual entropy satisfies

$$
k \ln g[l, \bar{s}(l)]-l S_{0} \approx \omega \bar{s}(l) \quad(l \rightarrow \infty) .
$$

The entropy per unit of surface $\omega$ is supposed finite but it could in principle be zero. (It 
might evidently, also, depend on temperature).

We may summarize these considerations by writing

$$
\ln G_{l}(\beta) \approx l\left(S_{0} / k\right)-\beta(w--T) \bar{s}(l)-T \ln l+\ln q_{0} \text {. }
$$

in which we have also specifically recognized the existence of higher order terms proportional to ln $l$ and of order unity. A logarithmic term was not included by the earlier workers and is not essential to our main considerations. However many studies of related combinatorial problems (in particular self-avoiding random walks) have shown that the asymptotic expansion should be expected to have this form with $T$ a positive number of magnitude unity or greater but depending principally on the dimensionality $[15,16]$.

Substituting with (30) and (20) into the expression (16) for the pressure, and adopting the form (27) purely for simplicity, yields finally

$$
p / k T=\pi(\beta, z)=q_{0} \sum_{l=1}^{\infty} l^{-\tau} x^{l \sigma} y^{l}
$$

where

$$
y=z \exp \left[\beta E_{0}+\left(S_{0} / k\right)\right] \text {, }
$$

and

$$
x=\exp \left[-a_{0} \beta(w-\omega T)\right] .
$$

so that $y$ is proportional to the activity and $x$ essentially measures the temperature (approaching zero as $T \rightarrow 0$ ). This completes the mathematical derivation of the theory on the basis of assumptions (A) and (B). Let us turn now to the consequences. to

Consider the probability of finding a cluster of size $l$. By (18) and (30) this is proportional

$$
\rho_{l}=q_{0} x^{\sigma} y^{l / l^{\top}}
$$

At low temperatures $x$ is small and at low activities (and hence low densities) $y$ is also small. In these circumstances $\rho_{l}$ decays rapidly to zero as $l$ increases (see Fig. 4). As $y$ approaches unity. however, the decay becomes slower. When $y=1, \rho_{l}$ still decays to zero but only as exp [-const. $\left.l \sigma\right]$. (assuming, of course, that $x<1$, that is, $\left.T<T_{c}=w / \omega\right)$. On the other hand when $y$ is slightly larger than unity the probability at first decreases because of the factor $x^{\sigma}$ but finally increases when the exponent $(\ln y) l$ dominates the exponent $-|\ln x| l^{\sigma}$ or, more generally, $\bar{s}(l)$ (see Fig. 4). The large (divergent) probability of very large clusters indicates, as observed before, that condensation has taken place. Consequently we identify $y=y_{\sigma}=1$ as the condensation point so. that

$$
\dot{z}_{\sigma}=\exp \left[-\beta\left(E_{0}-T S_{0}\right)\right] \text {. }
$$

while the chemical potential at condensation is

$$
\mu_{\sigma}(T)=-E_{0}-T S_{0}+\not / 2 k T d \ln \left(h^{2} / 2 \pi m k T\right) .
$$


If $y$ is only slightly greater than $y_{\sigma}$, that is if $\mu-\mu_{\sigma}(T)$ is very small, the minimum value of $\rho_{l}$ can be extremely small since

$$
l_{\text {min }} \simeq\left[a_{0}(w-\omega T) /\left(\mu-\mu_{\sigma}(T)\right]^{1 /(1-\sigma)}\right.
$$

will be very large. Consider a system in equilibrium at a chemical potential just less than $\mu_{\sigma}(T)$ in which $\mu$ is suddenly increased slightly (or, equivalently, in which $\mu_{\sigma}(T)$ is decreased slightly by cooling). Evidently even if the condensation point is passed the cluster distribution $P_{l}$ for $l<l_{\min }$ should scarcely have to change as the system "relaxes" to its new equilibrium. Furthermore if the primary mechanism by which a cluster can grow is through a binary collision with a relatively small cluster or single molecule (as will surely be the case at low densities) we see that there is a "free energy barrier" at $l=l_{\mathrm{min}}$; a cluster with a large value of $l$ less than $l_{\min }$ will tend to "evaporate" or break up into smaller clusters rather than grow further. Only occasional chance fluctuations, which will be extremely infrequent when $\mu-\mu_{\sigma}(T)$ (the "degree of supercooling") is small, will carry the system over the barrier. Even a single cluster with $l>l_{\min }$ will, however, tend to grow, increasingly rapidly as $l$ increases, and thus consitutes a "nucleus" of the condensed phase. (Impurities, such as dust particles,

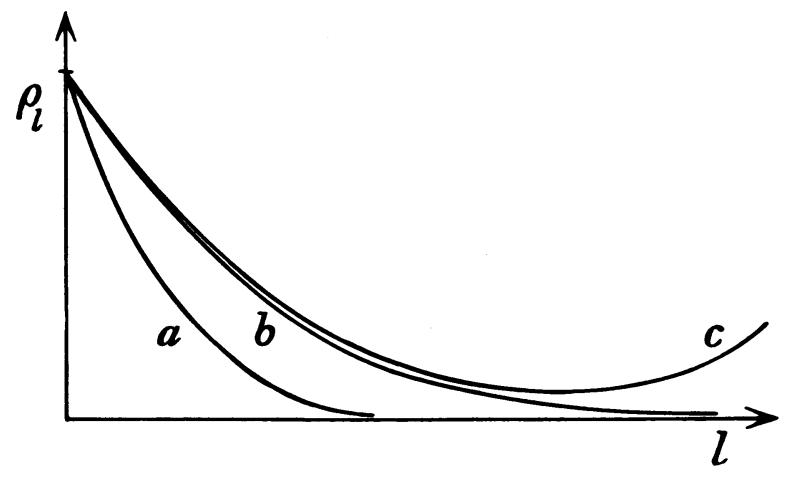

FIGURE 4

Density of clusters of size $l$ at an activity (a) below the condensation point, $y<1$, (b) at the condensation point, $y=1$, and (c) just above condensation at $y>1$.

may fulfill a similar role.) We can thus understand why a rather long lived metastable state may be observed when a pure gas is slightly supercooled. The extreme "limit of metastability" might be defined, somewhat arbitrarily, by the condition $l_{\min } \simeq 1$, which yields

$$
\left|\mu-\mu_{\sigma}(T)\right|_{\max } \simeq a_{0}(w-\omega T) \text {. }
$$

Evidently metastability, and in fact condensation, cannot occur if the temperature exceeds

$$
T_{c}=w / \omega
$$

which may thus be identified as the critical temperature. (Recall that $\omega$ might depend to some extent on temperature). 
Apart from certain refinements and generalizations we have so far essentially reproduced the droplet theory of condensation (and metastability!) as set out, for example, by Frenkel in his book [8]. Let us now enquire into the analytic properties of the theory with Mayer's conjecture in mind.

\section{Analytic Character and the Critical Point}

For fixed $x$, that is fixed temperature, the radius of convergence of the series (31) is given by

$$
y_{0}=\lim _{l \rightarrow \infty}\left|l^{-\top} x^{l \sigma}\right|^{-1 / l}=\lim _{l \rightarrow \infty}|x|^{-1 / l-\sigma}=1 \text {. }
$$

for all $x$. The last equality follows from the assumption $\sigma<1$. More generally the same result follows for any $\bar{s}(l, \beta)$ from the assumption (B $i)$. Since the terms in (31) are positive the point $y_{0}=1$ must be a singularity of the function. Furthermore we see that it coincides $w$ ith the previously identified condensation point $y_{\sigma}=1$. For this model, therefore Mayer's conjecture is verified: the condensation activity $z_{\sigma} \underline{\text { is }}$ a singularity of the analytic function $p(z) / k T$.

What is the behaviour of the density, the compressibility etc. at the condensation point? To answer this question note that these variables can be expressed in terms of the derivatives

$$
\pi^{(n)}(\beta, z)=\left(z \frac{\partial}{\partial z}\right) \pi=\left(y \frac{\partial}{\partial y}\right) \pi=q_{0} \sum_{l=1}^{\infty} l^{n-\tau} x^{l^{\sigma}} y^{l} .
$$

Thus the density and compressibility are given by

$$
\rho(\beta, z)=\pi^{(1)}(\beta, z), k T \rho^{2} K_{T}=\pi^{(2)}(\beta, z) .
$$

(The energy, specific heat, etc. may similarly be expressed in terms of derivatives with respect to $x_{\text {. }}$ ) At the condensation point we find

$$
\pi_{\sigma}^{(n)}(\beta)=\pi(n)\left(\beta, z_{\sigma}\right)=q_{0} \sum_{l=1}^{\infty} l^{n-\tau} x^{l^{\sigma}} .
$$

Using the assumption $\sigma>0$ or more generally (B ii), it is not difficult to show that this series converges for all $n$ provided only that

$$
x<x_{c}=1 \text {, that is, } T<T_{c}=w / \omega .
$$

(For $x>1$ the series (43) is evidently always divergent.) Thus, in particular, the compressibility remains finite on the condensation curve up to a temperature $T=T_{c}$ which may hence be identified as the critical point in agreement with our previous conclusion.

The fact that all the derivatives with respect to $z$ (for real $z$ ) remain finite at $z=z_{\sigma}$ even though we have established that this is a singularity of $\pi(z)$ might seem surprising at first sight. It merely means, however, that the singularity at $z=z_{\sigma}$ is an essential singularity. (One may recall the function $\exp \left(-1 / x^{2}\right)$ for which all derivatives along the real axis vanish 
at $x=0$ ). Evidently such a singularity can hardly be detected by direct thermodynamic measurements in the homogeneous phase since none of the thermodynamic functions will exhibit any infinities or similar "anomalies"! [17]

Since $\pi(z)$ has an essential singularity at $z=z_{\sigma}$ it is impossible to analytically continue the function through $z_{\sigma}$ to find some real "metastable continuation" of the isotherm. This may be seen clearly if we estimate the derivatives at $z_{\sigma}$ by approximating the sum in (43) by an integral, which is valid for $x$ near unity. Thus for $x<1$ and $n>t-1$ we have

$$
\pi_{\sigma}^{(n)}(\beta) \approx q_{0} \int_{0}^{\infty} l^{n-\tau} \exp \left[-\left(\ln x^{-1}\right) l \sigma\right] d l
$$

and making the substitution $t=\theta l \sigma$, where

$$
\theta=\ln x-1=\frac{a_{0} \omega}{k T}\left(T_{c}-T\right)
$$

yields

$$
\pi_{\sigma}^{(n)}(\beta) \approx\left(q_{0} / \sigma \theta(n-T+1) / \sigma\right) \Gamma\left(\frac{n-T+1}{\sigma}\right) .
$$

For large $n$ the gamma function varies as $(n !)^{1 / \sigma}$. Consequently if the derivatives are used to construct a Taylor series expansion about $z_{\sigma}$ in powers of (ln $\left.z-\ln z_{\sigma}\right)$ the coefficient of the $n$-th term will vary as $(n !)(1-\sigma) / \sigma$ for large $n$. Since, by assumption, $\sigma$ is less than unity and since $(n !)^{\varepsilon}$ diverges faster than $\zeta^{n}$ for any $\zeta$ when $\varepsilon$ is positive it follows that the Taylor series will never converge; that is, it has a zero radius of convergence. (For $z<z_{\sigma}$ and low enough $T$ it might, however, have an asymptotic character.)

Although one cannot find a real analytical continuation of $\pi(z)$ through $z=z_{\sigma}$ one may hope to construct a continuation of $\pi(z)$ by passing around the singularity at $z_{\sigma}$. This task is considered in Appendix B where it, is shown that $\pi(z)$ as defined by the expansion (31) extends into a function meromorphic in the entire complex $z$ plane except for a cut along the real $z$ axis from $z=z_{\sigma}$ to $\infty$. Across this cut the imaginary part of $\pi(z)$ has a discontinuity which varies as

$$
\pi(z+i \varepsilon)-\pi(z-i \varepsilon) \approx C\left(z-z_{\sigma}\right)^{\top *} e^{-D /(z-z)^{\sigma *}}, \quad(z \mathrm{real})
$$

when $z=\mathscr{R}\{z\} \rightarrow z_{\sigma}+$, where $C$ and $D$ are constants and

$$
\sigma^{*}=\sigma /(1-\sigma), \quad T^{*}=(\tau-1+1 / 2 \sigma) /(1-\sigma) \text {. }
$$

Evidently the discontinuity and all its derivatives vanish as $z \rightarrow z_{\sigma}$ along the cut, which accounts for the extreme weakness of the singularity.

Our analysis thus shows that the cluster model of condensation which, as we have argued, should be valid at low densities (and hence low temperatures) implies a singularity at the condensation point and hence the nonexistence of a well defined real isotherm beyond condensation. Although one can be less confident of the correctness of the assumptions at higher temperatures it is of interest to pursue the consequences of the model in the vicinity of its critical point. We may hope to throw some light on the general theory of critical phenomena and on Mayer's well known hypothesis of some sort of extended critical region often referred to as the "Derby hat" phenomena. 
Firstly note that from (41) and (42) the critical point density will be

$$
\rho_{c}=q_{0} \sum_{l=1}^{\infty} l 1-\tau
$$

and for this to be finite we must have $\tau>2$. From (42), (46) and (47) we see that provided $\tau<3$ the compressibility at condensation diverges to infinity (as expected) like $\left(T_{c}-T\right) \gamma^{\prime}$ with

$$
\gamma^{\prime}=(3-T) / \sigma .
$$

(The notation $\gamma^{\prime}$ for this exponent follows the standard scheme [18].) Evidently the nature of the critical point singularities in other variables will equally depend only on the two parameters $\sigma$ and $T$. Thus the shape of the gaseous side of the "coexistence curve" is found to be

$$
\rho_{c}-\rho_{\sigma}(T) \sim\left(T_{c}-\cdot T\right)^{\beta}
$$

where

$$
\beta=(\tau-2) / \sigma \text {. }
$$

The specific heat at constant critical density, which derives from $p_{\sigma}(T)$, diverges with an exponent

$$
\alpha^{\prime}=2-(\tau-1) / \sigma
$$

where a value $\alpha^{\prime}=0$ must be interpreted as meaning a logarithmic law rather than a power law.

The shape of the critical isotherm is given by

$$
p_{c}-p \sim\left(p_{c}-\rho\right) \delta
$$

where, by putting $x=1$ in (31), one easily finds

$$
\delta=1 /(\tau-2) \text {. }
$$

By a more complicated analysis one may also investigate the behaviour of the isotherms as the critical point is approached from above at constant density $\rho_{c}$. One discovers that the specific heat and compressibility exponents $\alpha$ and $\gamma$ above $T_{c}$, satisfy the symmetry relations

$$
\alpha=\alpha^{\prime}, \quad \gamma=\gamma^{\prime} .
$$

The general appearance of the isotherms implied by the cluster model when $2<\tau<3$ is sketched in Fig. 5. Apart from the absence of the "liquid" sections of the isotherms the overall behaviour is surprisingly like that of real fluids.

Since all the critical point exponents depend on only two parameters any three of them must be related. Thus we find, for example [19],

$$
\alpha^{\prime}+2 \beta+\gamma^{\prime}=2
$$

and

$$
\gamma^{\prime}=\beta(\delta-1) .
$$

These and other similar relations are satisfied exactly by the two-dimensional lattice gas with 
nearest neighbour interactions (Ising model) and, as far as the values are known accurately, by the three-dimensional model also [20]. They seem to be valid for real fluids although at present the experimental data are not sufficiently precise for a stringent test. Interestingly they hold also for a van der Waals-like gas which may be regarded as an infinite-dimensional system.

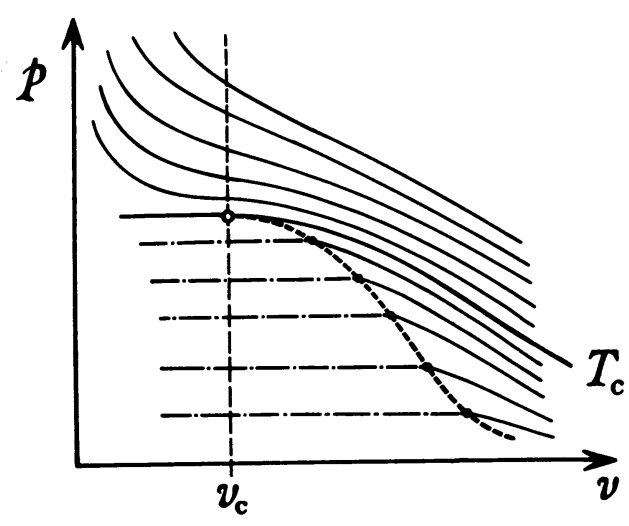

FIGURE 5

\begin{abstract}
Isotherms following from the cluster theory of condensation showing the gaseous side of the coexistence curve and the critical point.
\end{abstract}

of course using known values for $\gamma, \delta$ and $\beta$ etc. we may evaluate the parameters $\sigma$ and $\tau$ and compare them with our expectations. For the plane Ising model we find from

$$
\sigma=1 / \delta \beta=1 /\left(\gamma^{\prime}+\beta\right) \text { and } \tau=2+(1 / \delta)=2+\beta /\left(\gamma^{\prime}+\beta\right)
$$

the values

$$
\sigma=\frac{8}{1.5}=0.53 \dot{3} \text { and } T=2 \frac{1}{15} .
$$

This value of $\sigma$ is surprisingly close to the value $\sigma=1 / 2$ expected at low temperatures on naive geometrical grounds and tends to increase one's confidence in the underlying cluster picture. Presumably the difference $\Delta \sigma=0.03 \dot{3}$ is due to the neglected effects of excluded volume and to the statistical geometry of noncompact clusters. For the three-dimensional lattice gas there is some uncertainty due to lack of precise knowledge of the critical exponents. Present information is consistent with the ranges

$$
\sigma=\frac{16}{25}=0.640, \quad T=2 \frac{1}{5}
$$

to

$$
\sigma=\frac{8}{13}=0.6153 \ldots \quad T=2 \frac{5}{26}=2.192 \ldots
$$

These values of $\sigma$ fall below the geometrical value $\sigma=2 / 3$ but only by $\Delta \sigma \simeq 0.027$ to 0.051 .

This is, however, a sure indication of the importance of the excluded volume effects since single cluster geometrical factors would be expected only to increase $\sigma$. The "classical" or 
van der Waals-like limit corresponds to

$$
\sigma=\frac{2}{3} \quad \text { and } \quad T=2 \frac{1}{3} .
$$

This seems difficult to understand in a simple fashion in view of the expectation $\sigma \simeq 1$ for $d \rightarrow \infty$, and may be an indication of a more thoroughgoing breakdown of the cluster picture in the critical region.

Finally note that in all these cases we have a unique critical point rather than a critical region. Reference back to equation (43) and the accompanying argument shows that this follows quite generally from the two assumptions ( $B$ iii) that the surface entropy is proportional to the surface energy ( $\omega=0$ would give $T_{c}=\infty$ ), and (B ii) that the mean surface of a cluster of $l$ atoms increases more rapidly than in $l$. Conversely it is easy to see that if the surface entropy increased faster than the surface energy, the effective surface tension would be negative at all temperatures; small clusters would have no stability and a sharp condensation could not occur. Secondly suppose the surface energy and entropy increased only as fast as in $l$. Then the convergence of the series (43), that is of the $n$-th derivatives with respect to $z$ at condensation, would depend on the order of the derivative considered. This in turn would mean that the temperatures at which a volume discontinuity appeared, at which the compressibility became infinite, etc. would all be different. In other words the critical phenomena would take place over a range of different temperatures rather as suggested by Mayer. That this does not happen in practice is thus a reflection of the fact that the geometrical surface of a cluster of $l$ particles (in two or more dimensions) increases faster than ln $l$ (and probably as $l^{\sigma}$ with $\sigma<1$ ). A fuller description of this type of anomalous critical region will be given in the following sections in connection with an exactly soluble model displaying condensation to which we now turn.

\section{An Exactly Soluble Model}

As we have shown the cluster theory of condensation rests mainly on two assumptions: (A) that the interactions (in the form of excluded volume) between clusters can be neglected and (B) that the mean surface of a cluster has the expected properties and characterizes sufficiently well all clusters. In my opinion the second assumption is probably the more difficult one to justify? To judge the validity of the first assumption we will describe a one-dimensional model in which the excluded volume may be rigorously taken into account.

It is sometimes asserted that a one-dimensional model cannot display a phase transition (excluding, that is, the procedure of taking some special limit after the thermodynamic limit as in the Kac-Uhl enbeck-Hemmer model [3]). This has only been justified, however, for systems with pair interactions of strictly finite range $b$ and with similar three-body and many-body interactions up to some finite order; that is, all the forces vanish identically for separations greater than $b[21]$. Indeed it seems likely that a one-dimensional fluid of particles interacting with a pair potential decaying only as $1 / r^{\varepsilon}$ will exhibit condensation if $1<\varepsilon<2$. This is suggested by the cluster argument, as we will outline, and also, as has been remarked by Kac [22], because the corresponding "sphericalized" lattice gas model still has a transition whereas the normal tendency of "sphericalization" seems to be to destroy transitions. At present, however, no such pair interaction model has been rigorously solved. We will consider instead a model in which the forces are of strictly finite range but many-body interactions of indefinitely great order are present. By strictly finite range we mean, as above, that if any group 
of $N_{1}+N_{2}$ particles with total potential energy $U_{N_{1}}+N_{2}$ is separated into a group of $N_{1}$ particles and a group of $N_{2}$ particles with a minimum separation $R$ between particles in different groups exceeding $b$, then there is no mutual interaction, between the groups, that is

$$
\Phi_{N_{1}, N_{2}}(R)=U_{N_{1}+N_{2}}-\left(U_{N_{1}}+U_{N_{2}}\right) \equiv 0, \quad R>b .
$$

Explicitly we take a pair interaction potential

$$
\begin{array}{rlrl}
U^{(2)}(r)=\phi_{2}(r) & =+\infty, \quad r \leqslant a, \\
& \geqslant-w_{A}, \quad a<r \leqslant b . \\
& =0, & & r \geqslant b .
\end{array}
$$

so that the particles have a hard core of diameter $a$. We suppose for simplicity that the range satisfies

$$
b \leqslant 2 a
$$

so that the pair interactions arise only between nearest neighbours. In the interval $a<r \leqslant b$ the potential may be arbitrary provided it is bounded below. To define the many-body interactions we introduce, as in Section 3, a clustering distance $c$ which might be taken equal to $b$ although this is not necessary. Labelling the particles in sequence we say that particles $j$ to $j+k$ belong to the same cluster if $\left|r_{j+1}-r_{j}\right| \leqslant c,\left|r_{j+2}-r_{j+1}\right| \leqslant c, \ldots$ $\left|r_{j+k}-r_{j+k-1}\right| \leqslant c$. The $s$-body potential is then taken as $(s \geqslant 3)$

$$
\begin{aligned}
U(s)\left(r_{1}, \ldots r_{s}\right) & =\Phi_{s}, \text { if }\left|r_{i}-r_{j}\right| \leqslant c \\
& \text { for } s-1 \text { pairs } i=1, \ldots s \neq j=1, \ldots s . \\
& \equiv 0, \text { otherwise. }
\end{aligned}
$$

Thus there is a constant $s$-body interaction energy coming into play between any succession of $s$-particles which belong to the same cluster but no interaction between particles in different clusters. For simplicity we will assume the $\phi_{s}$ are all negative or zero (i.e. the many-body forces are attractive).

Consider the total energy of an (isolated) cluster of $l$ particles. This will be

$$
\begin{aligned}
E_{l} & =\sum_{i=1}^{l-1} \Phi_{2}\left(r_{i+1}-r_{i}\right)+(l-2) \phi_{3}+(l-3) \phi_{4}+\ldots+\phi_{l} . \\
& =\sum_{i=1}^{l-1} \Phi_{2}\left(r_{i+1}-r_{i}\right)+\sum_{s=3}^{l}(l+1-s) \phi_{s} .
\end{aligned}
$$


Evidently the energy per particle in an infinite cluster satisfies

$$
e_{\infty} \geqslant-w_{A}+\sum_{3=3}^{\infty} \Phi_{s}
$$

For thermodynamic stability we must require [5] $e_{\infty}>-\infty$ or

$$
\Phi=\sum_{s=3}^{\infty}\left(-\Phi_{s}\right)<\infty
$$

so that $\left|\phi_{s}\right|$ must decrease faster than $1 / s$. It should perhaps be stressed that the condition (71) together with the strictly finite range property (65), is sufficient to guarantee rigorously the existence of a limiting free energy with the usual thermodynamic properties even when $\varphi_{s}$ does not vanish for any $s[5]$.

Now we may rewrite (69) in the form

$$
\begin{aligned}
E_{l} & =-l \Phi+\sum_{i=1}^{l-1} \Phi_{2}\left(r_{i+1}-r_{i}\right)+w_{l} \\
& \geqslant-l\left(\Phi+w_{A}\right)+w_{l}+w_{A} .
\end{aligned}
$$

where the first term is recognised as a bulk energy while the surface energy is (neglecting a constant contribution)

$$
w_{l}=W(l)=\sum_{t=1} \sum_{s=t+1}^{\infty}\left(-\Phi_{s}\right)=\sum_{s=3}^{\infty} \min \{s-1, l\}\left(-\varphi_{s}\right) .
$$

It is easy to show that

$$
W_{l} / l \rightarrow 0 \text { as } l \rightarrow \infty
$$

as expected [compare with (25)].

Notice at this point that if, in place of the many-body potentials, we had considered longrange pair interactions, we would have found precisely the same results (69) to (75) for the energy of an isolated cluster of $l$ particles of uniform spacing $d$ but with $\phi_{s}$ replaced by $\phi_{2}[(s-1) d]$. Thus our many-body forces accurately imitate the effects of long-range pair interactions within a single cluster. They do not, however, reproduce the long-range attractive forces between different clusters that arise in the pair case; neglect of these would, of course, be expected to weaken any tendency towards condensation. On the other hand the repulsions, or excluded volume effects, between different clusters are given precisely by our potentials.

If we accepted the arguments of the droplet theory of condensation we would conclude from 
(72) and (75) that the model would display condensation provided that

$$
W_{l} / \ln l \rightarrow \infty \quad \text { as } \quad l \rightarrow \infty .
$$

There should then be no critical point since there is evidently no surface contribution to the entropy of one-dimensional clusters (except perhaps for a constant contribution from the ends of the cluster). Consequently the entropy per unit surface, $\omega$ would vanish and by (44) we would have $T_{c}=\infty$. If $W_{l}$ varied as $\ln l$ for large $l$ we would expect the Derby-hat type of phenomena while if, on the other hand,

$$
W_{l} / \ln l \rightarrow 0 \text { as } \quad l \rightarrow \infty
$$

there would be no phase transition. As we will show these conclusions are confirmed in detail by the exact solution of the model!

Before describing the solution note that from (73) we obtain

$$
\varphi_{s}=W_{s}-2 W_{s-1}+W_{s-2}
$$

so that the conditions (75) and (76) become

$$
s \varphi_{s} \rightarrow 0, \quad \text { as } \quad s \rightarrow \infty
$$

and

$$
s^{2} \varphi_{s} \rightarrow \infty \text {, as } s \rightarrow \infty \text {. }
$$

respectively. For potentials decaying faster than $1 / s^{2}$ no transition is thus expected.

\section{Analysis of the Model}

We will sketch, without entering into full details, the solution of the model with the potentials (66) to (68). As with most one-dimensional models it is advantageous to start with the grand partition function $\Xi(\beta, z ; L)$ for a length $L$ and to compute its Laplace transform

$$
\Psi(\beta, z, s)=\int_{0}^{\infty} e^{-s L} \equiv(\beta, z, L) d L,
$$

which can be regarded as a generating function for all possible sets of clusters of all possible sizes and spacings. If we write

$$
J(\beta, s)=\int_{0}^{c} e^{-s r} e^{-\beta \phi(r)} d r
$$

and

$$
K(\beta, s)=\int_{c}^{\infty} e^{-s r} e^{-\beta \phi(r)} d r
$$

and define the generating function 


$$
H(\beta, z, s)=\sum_{l=1}^{\infty} z^{l} e^{-l \beta \Phi}[J(\beta, s)]^{l-1} e^{-\beta W(l)} .
$$

which enumerates all possible single clusters with their "internal" Boltzmann factors, we can construct $\Psi(\beta, z, s)$ from the series

$$
\Psi(\beta, z, s)=s^{-1}+s^{-1} H s^{-1}+s^{-1} H K H s^{-1}+s^{-1} H K H K H s^{-1}+\ldots
$$

Here the first term accounts for all lengths of line with no clusters, the second term for all possible single clusters at all positions along the line, the third term for all pairs of clusters and so on. The solution of the problem is thus given formally by

$$
\Psi(\beta, z, s)=s^{-1}+s^{-2} \sum_{m=1}^{\infty}[H(\beta, z, s)]^{m}[K(\beta, s)]^{m-1} .
$$

The thermodynamic behaviour is obtained by noting, from the definition (81), that the abscissa of convergence, $s_{0}=s_{0}(\beta, z)$, of the transform $\Psi(s)$ determines the grand canonical potential since

$$
\beta p=\pi(\beta, z)=\lim _{L \rightarrow \infty}(1 / L) \text { ln } \equiv(\beta, z, L)=s_{0}(\beta, z) .
$$

The possibility of a phase transition may be seen immediately since, from (84) and (86), the breakdown of the convergence of $\Psi(s)$ for small $\mathscr{R}\{s\}$ is determined either by the

Interior condition

$$
z e^{-\beta \Phi} J(\beta, s)=u(\beta, z, s)=1 \text {, }
$$

or by the

Exterior condition

$$
H(\beta, z, s) K(\beta, s)=1
$$

If, as $s$ decreases from $\infty$, one of these conditions is always encountered before the other there is no phase transition; a change over from one condition to the other will correspond to some sort of phase change.

The second condition can be conveniently rewritten in terms of the "master function"

$$
\Upsilon(\beta, u)=\sum_{l=1}^{\infty} u^{l} e^{-\beta W(l)}
$$

defined for $|u|<1$, which bears an obvious resemblance to the final form (31) of the grand potential in the droplet theory. In particular notice that $Y(\beta, u)$ is always singular at $u=1$. The exterior condition then becomes

$$
Y(\beta, u)=J(\beta, s) / K(\beta, s)=Q(\beta, s)
$$


where the function $Q(\beta, s)$ is easily shown to increase monotonically (and strictly) in $s$ from the value $Q(\beta, 0)=0$.

Since $s_{0}$ is only determined implicitly by the equations it is simpler to choose $s=\beta p$ as an independent variable and to ask for the activity (or chemical potential or Gibbs free energy) as a function of $\beta$ and $s$, that is, $T$ and $p$. One readily finds from (88)

$$
\ln z(\beta, s)=\ln u(\beta, s)-\ln J(\beta, s)+\beta \Phi
$$

and the equation of state is then

$$
v=v(\beta, s)=(\partial / \partial s) \text { ln } u(\beta, s)-(\partial / \partial s) \text { ln } J(\beta, s) \cdot
$$

Now for small $s=\beta p$ the function $u(\beta, s)$ is determined through (91), the series (90) converging absolutely. As $s$ increases $u$ increases analytically towards unity. If the series (90) diverges at $u=1$, that is if

$$
\Upsilon(\beta, 1-)=\sum_{l=1}^{\infty} e^{-\beta W(l)}=\infty .
$$

one easily sees that $u(\beta, s)$ remains less than unity for all $s$ (i.e. all $p$ ) and there is no transition. If, one the other hand, $X(\beta, 1-)$ is finite then a transition occurs at a pressure $\beta p_{\sigma}=s_{\sigma}$ determined by

$$
Q\left(\beta, s_{\sigma}\right)=\gamma(\beta, 1-)
$$

For $s \geqslant s_{\sigma}$ the function $u$ has the constant value unity so that, by (92) the thermodynamic behaviour is governed entirely by the "internal function" $J(\beta, s)$.

We have thus found the conditions for a phase transition in our model. By studying the convergence of the series (94) it is not difficult to show there is

(a) no transition at any temperature

$$
W_{l} / \ln l \rightarrow 0(l \rightarrow \infty) \text { or }-s^{2} \Phi_{s} \rightarrow 0 \quad(s \rightarrow \infty) ;
$$

(b) a transition at all temperatures

$$
W_{l} / \ln l \rightarrow \infty \quad(l \rightarrow \infty) \text { or }-s^{2} \varphi_{s} \rightarrow \infty \quad(s \rightarrow \infty) ;
$$

(c) a transition below a critical temperature

if $W_{l} / \ln l$ or $-s^{2} \Phi_{s}$ approach nonzero limits.

These results agree with the guesses based on the droplet theory. They may be summarized conveniently by the formula for the critical temperature, namely,

$$
k T_{c}=\lim _{l \rightarrow \infty} W_{l} / \ln l \text { or } \underset{s \rightarrow \infty}{\lim }-s^{2} \Phi_{s} .
$$

since cases (a) and (b) correspond merely to $T_{c}=\infty$ or $T_{c}=0$, respectively.

The nature of the transition when it occurs depends, of course, on further details of $W_{l}$. 
From (93) the volume discontinuity at the transition is seen to be

$$
\Delta v=Q^{\prime}\left(\beta, s_{\sigma}\right) / Y_{1}(\beta, 1)
$$

where $Q^{\prime}(s)=\partial Q / \partial s>0$ and the derivatives of the master function are defined by

$$
Y_{k}(\beta, u)=\sum_{l=1}^{\infty} l k u^{l} e^{-\beta W(l)} .
$$

In case (b), which corresponds to the behaviour of surface energy expected in two or more dimensions, there is always a volume discontinuity at the transition although it diminishes as the pressure increases. On the other hand the compressibility and all higher derivatives along the isotherm remain finite as the pressure increases towards $p_{\sigma}$ (since all the series (101) converge at $u=1$ ). Nevertheless it is clear that the condensation point is a singular point of the isotherm, as Mayer conjectured although, as we would have anticipated, it is an essential singularity. This leading conclusion of the cluster theory is thus not invalidated by the full inclusion of the excluded volume effects - at least not in this simple but rigorous model!

The absence of a critical temperature in the case (b) stems as we mentioned it would, directly from the absence in the expression for the master function of any entropy factors with the same behaviour as $W_{l}$. We could at the cost of no longer having a model with a definite Hamiltonian, arbitrarily "assign" extra phase space to each cluster of $l$ particles in such a way as to lead to an additional factor $\exp \left[(\omega / k w) W_{l}\right]$ in the $l$-th term of $Y(\beta, u)$. We would then find a unique critical point at a temperature $T_{c}=w / \omega$, just as in the cluster theory except that the model now also describes the liquid phase. Apart from the liquid side of the coexistence curve (which is concave rather than convex due, essentially, to the neglect of "bubbles" in the liquid) the overall pattern of isotherms resembles that found in practice. In particular it is possible to pass continuously and smoothly from gas to liquid over the "top" of the critical point. This transition, however, is not fully analytic since one finds that the critical isotherm for $v<v_{c}$ is a line of essential singularity. Nothing of this is "visible", however, since all temperature or pressure derivatives are continuous through this isotherm! This means that in the model (no longer, we stress, a true Hamiltonian model) there is an absolute distinction between a gas and a liquid. Such a distinction does not, of course, occur with a van der Waals-like equation of state. The existence of some absolute difference between liquid and gas has often been conjectured for real systems but has never been established convincingly. If the nature of the transition between the states were of such an "infinite order" this is perhaps hardly surprizing. I do not feel, however, that our result adds much plausibility to the speculation for realistic models although it serves as a warning of what could happen!

Finally let us investigate briefly the borderline case (c) where the model with a proper Hamiltonian does have a critical temperature. The main features follow from the observation that the number of derivatives (101) of the master function which remain finite at the transition point $u=1$ depends on temperature. As soon as $T$ drops below $T_{c}$, as defined in (99), all sufficiently high derivatives diverge which means that high derivatives along the isotherm are discontinuous across a singular curve. in the $(p, v)$ plane. However there is no volume discontinuity until the pressure drops to $T_{c}{ }^{\prime}=y_{2} T_{c} !$ on the other hand the compressibility remains continuous across the singular curve only down to a temperature of $(2 / 3) T_{c}=(4 / 3) T_{c}^{\prime}$ below which point it becomes infinite as the transition is approached from the low density side. It remains infinite at the transition down to a temperature of $(1 / 3) T_{c}=(2 / 3) T_{c}^{\prime}$ but becomes finite again as in a normal condensation process, at lower temperatures! One might indeed say that the "order" of the transition varies continuously with temperature. 
The general shape of the isotherms when

$$
W_{l}=w \ln l+\frac{1}{2}(1-\lambda) \ln \ln l,(\lambda>0) \text {. }
$$

is illustrated in Fig. 6. In this case the critical pressure is infinite while the gas side of the coexistence curve varies as $\left(T_{c}{ }^{\prime}-T\right)^{\lambda}$ when $T$ approaches $T_{c}{ }^{\prime}$ from below. It is quite possible, however, to have a "flat-topped" coexistence curve as suggested originally by Rice [23].

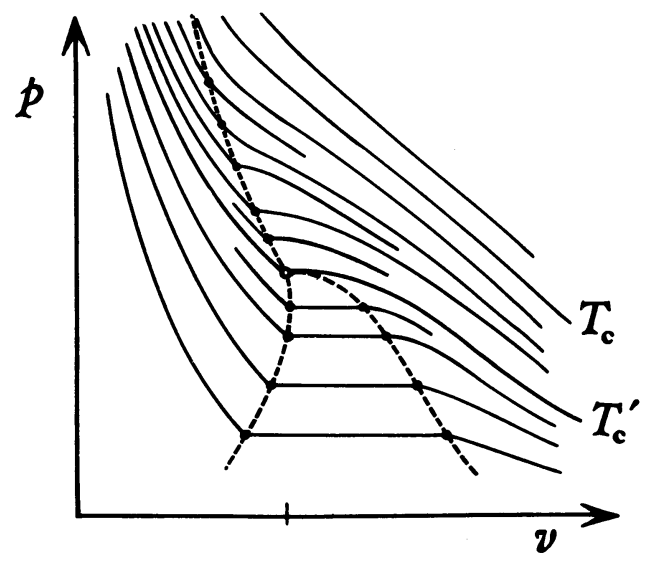

FIGURE 6

Sketch of the isotherms of the exactly soluble one-dimensional model in the borderline case (c) when the surface energy is given by equation (102).

This bewildering variety of peculiar possibilities is a further reflection of the artificially weak nature of the surfàce energy in case (c). Such a logarithmically increasing surface energy can arise, I believe, only in one dimension. Geometry alone will lead to surface energies satisfying (97) that is condition (B $i$ ) of Section 4, in two or more dimensions.

The one-dimensional model can be generalized in various directions (in particular, the liquid and gaseous states may be treated more symmetrically) but the description of these developments must await another occasion.

\section{Conclusions}

My primary purpose in this lecture has been to show that the physical ideas of the droplet theory of condensation still deserve further exploration. The theory itself contains interesting and, I believe basically correct, implications regarding the nature of the condensation point and the critical point which do not seem to have been noticed previously. The predictions of an essential singularity at the condensation point, of the uniqueness of the critical point and of the inter-relations between the critical point singularities throw light on a number of long standing problems and conjectures.

I hope by reformulating and extending the cluster theory I have exposed the most important 
problems concerning its foundations. The exactly soluble model which I described, although it is evidently artificial in a number of respects, lends support to the validity of the conclusions and underlying assumptions of the droplet theory. There seems to be a real possibility of establishing these foundations on a more rigorous basis. The subtle and complex possibilities already revealed by our analysis suggests that this may not be an easy task; it is, however, a worthwhile and important one.

\section{Acknowledgements}

The ideas described in this article have been developed over the course of a number of years. I have been greatly encouraged and stimulated in this period by Professor Mark Kac, and more recently, by Professor G.E. Uhlenbeck. It is a pleasure to thank them. I am also indebted to Professor N.G. van Kampen and Professor J.S. Langer for informative discussions in connection with Appendix B. The partial support of the National Science Foundation is gratefully acknowledged.

\section{APPENDIX A}

\section{Proof of Theorem on Condensation Singularities}

Suppose we are given, at fixed temperature, the pressure as a function of the specific volume $v$, that is,

$$
p=P(v)
$$

and we wish to determine the grand potential

$$
\beta p=\pi(\ln z)
$$

and the density

$$
p=1 / v=1 / v(\ln z)=\partial \pi / \partial \ln z
$$

as functions of the activity $z$. We may revert (A.3) in the form

$$
\ln z=L(v)
$$

and then solve formally for $\pi$ by integrating to obtain

$$
\pi(\ln z)=\int^{\ln z}\left[1 / L^{-1}\left(\ln z^{\prime}\right)\right] d \ln z^{\prime} .
$$

Conversely from (A. 3)

$$
1 / v=\beta(\partial P(v) / \partial v)(\partial v / \partial \ln z)
$$

so that solving for the last derivative and integrating yields

$$
\ln z-\ln z_{a}=\beta \int_{v_{a}}^{v} v^{\prime}\left(\frac{\partial P}{\partial v}\right) d v^{\prime}=\beta\left[p v-p_{a} v_{a}-\int_{v_{a}}^{v} P\left(v^{\prime}\right) d v^{\prime}\right] \text {. }
$$

which determines the function $L(v)$ upto an additive constant. 
This result may be checked directly by thermodynamics since, in standard notation,

$$
G_{N}=p V_{N}+F_{N}
$$

while the chemical potential is

$$
\begin{aligned}
\mu & =G_{N} / N=p v+F_{N} / N \\
& =p v-\int^{v} p\left(v^{\prime}\right) d v^{\prime}
\end{aligned}
$$

since

$$
p=-\left(\partial F_{N} / \partial V_{N}\right)=-\left(\partial\left[F_{N} / N\right] / \partial v\right)
$$

Recalling that ln $z=\beta \mu+$ constant shows the equivalence of (A.9) to (A.7).

Now suppose that $P(v)$ is analytic at $v=v_{\sigma} \neq 0$ so that the power series

$$
p=p_{\sigma}+\sum_{n=1}^{\infty} a_{n}\left(v-v_{\sigma}\right)^{n}
$$

is convergent for small enough $\left(v-v_{\sigma}\right)$. Substitution in (A.7) with $z_{a}=z_{\sigma}$ etc. yields the convergent series

$$
\ln z=L(v)=\ln z_{\sigma}+\beta \sum_{n=1}^{\infty} n^{-1}\left[n a_{n} v_{\sigma}+(n-1) a_{n-1}\right]\left(v-v_{\sigma}\right)^{n}
$$

where $a_{0} \equiv 0$. Thus $L(v)$ is analytic near $v=v_{\sigma}$.

Provided the coefficient of $\left(v-v_{\sigma}\right)$ in (A.12), namely $\beta a_{1} v_{\sigma}$, does not vanish, the function $L(v)$ may be reverted to yield $v$ as a function of $L=\ln z$, which is analytic in the neighbourhood of $L_{\sigma}=\ln z_{\sigma}$. The condition $a_{1} \neq 0$ is equivalent to the finiteness of $\partial v / \partial p$ and hence of the compressibility, at $v_{\sigma}$. Since $v_{\sigma} \neq 0$ the reciprocal is also analytic and consequently the integration in (A.5) yields $\pi(z)$ as an analytic function of $z$ near $z_{0}$.

If $a_{1}=a_{2}=\ldots=a_{k-1}=0$ and $a_{k} \neq 0$ so that the compressibility is infinite at the condensation point one sees similarly that $L^{-1}(\ln z)$ is analytic in the variable $\left(\ln z-\ln z_{\sigma}\right)^{1 / k}$ so that $\pi(z)$ has a simple branch point at $z=z_{\sigma}$.

\section{APPENDIX B}

\section{Analytical Continuation of Droplet Model Grand Potential}

From (31) to (33) and (46) the grand potential, $\pi=p / k T$, for the droplet model is given by

$$
\pi(z)=q_{0} \sum_{l=1}^{\infty} l^{-\tau} e^{-\theta l \sigma} y^{l}, \quad y=z / z_{\sigma} \text {. }
$$


Following van Kampen [24] we attempt to find a function $f(t)$ such that

$$
\pi(z)=\int_{0}^{\infty} \frac{y}{e^{t}-y} f(t) d t .
$$

Expanding formally in powers of $y$ under the integral sign one finds that $f(t)$ should satisfy

$$
\int_{0}^{\infty} e^{-l t} f(t) d t=q_{0} l-\tau e^{-\theta l \sigma} .
$$

Inverting this Laplace transform yields

$$
f(t)=\frac{1}{2 \pi i} \int_{c-i \infty}^{c+i \infty} q_{0} p^{-\tau} e^{-\theta p \sigma} e^{p t} d p
$$

for any $c>0$. For $\theta>0$ and $0<\sigma<1$ the integrand in (B.4) at $p=c+i s$ has the bound

$$
q_{0}\left(c^{2}+s^{2}\right)^{-\tau / 2} e^{-\theta|s| \sigma_{\cos }(\pi \sigma / 2)} e^{c t} \text {. }
$$

Consequently $f(t)$ is defined by (B.4) for all real $t$ and in turn has a bound of the form

$$
f(t)<A \mathrm{e}^{c t}, \quad A=A(c, \sigma, \tau, \theta) .
$$

Since $c$ may be chosen less than unity the integral in (B.2) exists for all $y$ except for $y=$ $\mathscr{R}\{y\} \geqslant 1$, and, in fact, defines a function of $y$ meromorphic in the cut plane.

One may now re-expand (B.2) in powers of $y$ and check that the remainder after $n$ terms, namely

$$
R_{n}(y)=y^{n+1} \int \frac{e^{-n t}}{e^{t}-y} f(t) d t,
$$

vanishes as $n \rightarrow \infty$ provided $|y|<1$. Thus the integral (B.2) does represent $\pi(z)$ as defined by the series expansion (B. 1). Furthermore it evidently continues $\pi(z)$ to the whole $z$ (or $y$ ) plane except for a cut along the real axis from $z=z_{\sigma}$ to $+\infty$. On the cut the real part of $\pi(z)$ is obtained by taking the principal value integral in (B.2). On the other hand the discontinuity of the imaginary part of $\pi(z)$ across the cut at $z=\zeta>z_{\sigma}$ is given by

$$
\pi(\zeta+i \varepsilon)-\pi(\zeta-i \varepsilon)=2 \pi i f\left[\ln \left(\zeta / z_{\sigma}\right)\right], \quad \varepsilon \rightarrow 0 .
$$

To evaluate this discontinuity as $\zeta \rightarrow z_{\sigma}+$ or $y \rightarrow 1^{+}$. we use the method of steepest descents to perform the Laplace inversion (B.4) for small $t$. Making the substitution $p=u / t$ brings the integrand to the form

$$
u^{-\tau} \exp [u-X u \sigma]
$$

where $X=\theta / t^{\sigma}$ is now the "large parameter". To sufficient approximation the saddle point is at

$$
u=u_{0}=(\sigma X) 1 /(1-\sigma)=(\sigma \theta)^{1 /(1-\sigma)_{t}}-\sigma /(1-\sigma) .
$$

Finally changing variables again by putting $u=u_{0}(1+i v)$ leaves, after removal of a constant 
factor, an integrand of the form

$$
(1+i v)^{-\top} \exp \left\{\left(\sigma^{-1}-1\right) u_{0}\left[(1+i v)^{\sigma}-1-\sigma i v\right]\right\} \approx \exp \left[-\not / 2\left(\sigma^{-1}-1\right) u_{0} v^{2}\right]
$$

which peaks sharply when $t \rightarrow 0$ so that $u_{0} \rightarrow \infty$. In all, we find as $t \rightarrow 0$,

$$
2 \pi f(t) \approx[2 \pi / \sigma(1-\sigma)]^{K /}(t / \sigma \theta)^{\top *} e^{-g / t^{*}}
$$

where

$$
\sigma^{*}=\sigma /(1-\sigma), \quad \tau^{*}=(\tau-1+\not / 2 \sigma) /(1-\sigma),
$$

and

$$
g=(1-\sigma) \sigma^{\sigma *} \theta^{1 /(1-\sigma)} \text {. }
$$

FOOTNOTES and REFERENCES

1. L. ONSAGER, Phys, Rev. 65, 117 (1944).

2. C.N. YANG and T.D. LEE, Phys. Rev. 87, 404 (1952).

3. M. KAC, G. E. UHLENBeCK and P.C. HEMmer, J. Math. Phys, 4, 216 (1963), See also G. A. BAKER, Jr. Phys. Rev. 122, 1477 (1961) and N.G. van KAMPEN Phys. Rev. 135, A362 (1964).

4. This result has been proven recently with great generality by J.L. LEBOWITZ and 0. PENROSE, J. Math. Phys. 7, 98 (1966) and E. LIEB, J. Math. Phys. 7, 1016 (1966).

5. See, for example, M.E. FISHẸR, Archs ration. Mech. Analysis 17, 377 (1944).

6. See the account in Mayer's book: J.E. MAYER and M.G. MAYER, Statistical Mechanics, Chapter 14. John Wiley, New York (1940).

7. S. KATSURA, Adv. Phys. 12, (48) 391 (1963). In this connection reference should also be made to the work of: K. IKEDA, Prog. theor. Phys., Osaka 11, 336 (1954); 19, 653 (1958); 16, 341 (1956); 26, 173 (1961).

8. J. FRENKEL, J. Chem. Phys. 7, 200 (1939): letter to the Editor dated November 1938; ibid 7, 538; Kinetic Theory of Liquids, Chap. 7. Oxford University Press (1946).

9. W. BAND, J. Chem. Phys. 7, 324 (1939): paper received 6th March 1939; ibid 7, 927 (1939).

10. A. BIJL, Discontinuities in the Energy and Specific Heat, Doctoral Dissertation, Leiden, presented 29th April 1938.

11. R. Peierls, Proc. Camb. phil. Soc. 32, 477 (1936).

12. R.B. GRIFFITHS, Phys. Rev, 136, A437 (1964). 
13. J. DE BOER. Theorie de la Condensation in Changements de Phases, Comptes Rendus $2^{e}$ Reunion de Chimie Physique, Paris (1952).

14. In this connection it is interesting that B.J. HILEY and M.F. SYKES, J. Chem. Phys. 34, 1531 (1961) have shown by numerical, studies that for polygons of length $s$ and area $l$ on the plane triangular and square lattices the mean area appears to $\operatorname{vary}$ as $\langle l\rangle_{s} \sim{ }_{s}$ with $\theta=1.50 \pm 0.04$. This corresponds roughly to $\sigma=2 / 3$.

15. This term was introduced in J.W. ESSAM and M. E. FISHER, J. Chem. Phys, 38, 802 (1963), who demonstrated its significance in the critical region (see below).

16. See, for example, M.E. FISHER and M.F. SYKES, Phys. Rev. 114, 45 (1959) and later papers.

17. The conclusion that the condensation point should be an essential singularity of the activity series was advanced at the I.U.P.A.P. Conference on Phase Transitions at Brown University in June 1962 and was reported briefly by Katsura (on page 416 of the reference of Footnote 7) and in the 1964 University of Colorado, Boulder, Lectures in Theoretical Physics. More recently A.F. ANDREEV, Soviet Phys. JETP 18, 1415 (1964) has reached the same conclusion. It should also be mentioned that some of our analysis and conclusions have been closely foreshadowed in an interesting but apparently little appreciated paper by J.E. MAYER and S. F. STREeTER, J. Chem. Phys. 7, 1019 (1939).

18. See the reference in Footnote 15 and M.E. FISHER, J. Math. Phys. 5, 944 (1964).

19. The equality (58) was first advanced in Reference 15. Without entering into details here it should be mentioned that (59) was proposed independently by B. WIDOM, J. Chem. Phys. 41, 1633 (1964). Furthermore G.S. RUSHBROOKE, J. Chem. Phys. 39, 842 (1963), has proved that the inequality obtained from $(58)$ by replacing $=$ by $\geqslant$ is a thermodynamic necessity and R. B. GRIFfiths, Phys. Rev. Lett. 14, 623 (1965) has shown the same is true of the corresponding inequality obtained by substituting for $\gamma^{\prime}$ with (59).

20. D.S. GAUNT, M.E. FISHER, M.F. SYKES and J.W. ESSAM, Phys. Rev. Lett. 13, 713 (1964). For a more recent assessment see M.E. FISHER, J. appl. Phys. 38, 981 (1967) and Rep. Prog. Phys. 30,(1967).

21. L. VAN hOVE, Physica 16, 137 (1950).

22. At the I.U.P.A.P. Conference on Phase Transitions at Brown University, June 1962.

23. O.K. RICE, J. Chem. Phys, 15, 314 (1947); J. phys. Colloid Chem. 54, 1293 (1950). See also B. H. ZIMM, J. phys. Colloid Chem. 54, 1306 (1950).

24. N.G. van KAMPEN, private communication. 